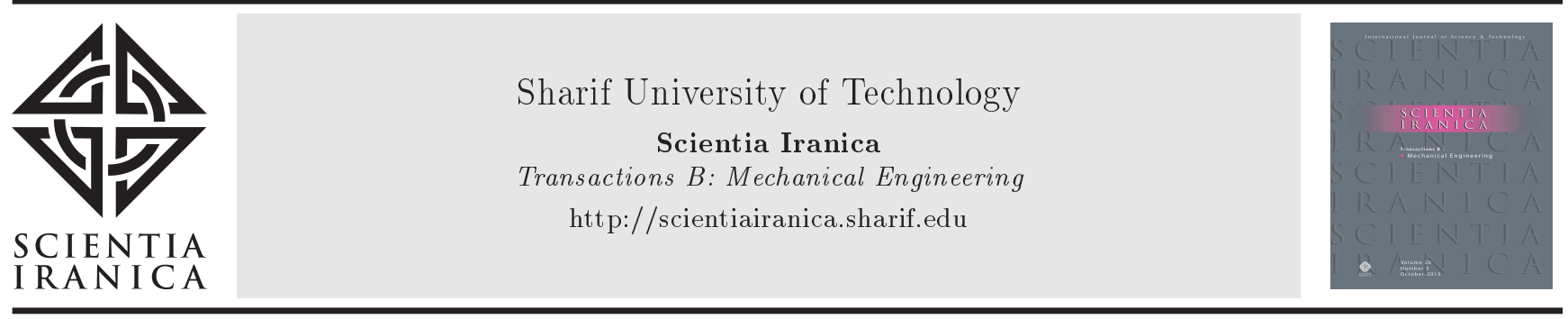

Research Note

\title{
A new algorithm to solve sinusoidal steady-state Maxwell's equations on unstructured grids
}

\author{
S. Azimi and M.S. Saidi* \\ School of Mechanical Engineering, Sharif University of Technology, Tehran, Iran.
}

Received 28 September 2015; received in revised form 13 November 2016; accepted 31 May 2017

\section{KEYWORDS}

Maxwell's equations;

Numerical solution;

Unstructured grid;

Yee's method;

Enclosed volume

\begin{abstract}
A new approach for attaining numerical solution to sinusoidal steady-state Maxwell's equations is developed. This approach is based on Yee's method, and can be applied on unstructured grids. A problem is solved by the method and the results show good agreement with the available analytical solution. This method can be improved to be applicable for general unsteady problems.
\end{abstract}

(C) 2018 Sharif University of Technology. All rights reserved.

\section{Introduction}

The first method to numerically solve Maxwell's equations was Finite Difference Method (FDM) developed in 1966 by Yee [1]. Because of its simplicity, FDM has been widely used and modified over time. For instance, Taflove developed FDM for steady-state sinusoidal problems [2], and Taflove et al. applied FDM to problems involving geometrically complicated radar surfaces [3]. Generally, the finite difference method can be applied to structured grids that can be constructed in simple geometries very well, but their construction in complicated geometries will usually result in high numerical errors [4]. Therefore, in these geometries, unstructured grids should be constructed.

There are several methods to solve Maxwell's equations on unstructured grids. Finite Volume Method (FVM) is one of the methods developed in 1995 by Shang [5]. This method is being widely used to solve Maxwell's equations. For instance, Jahandari et

\footnotetext{
*. Corresponding author. Tel.: +98 2166165558 ; Fax: +98216600 0021

E-mail address: mssaidi@sharif.edu (M.S. Saidi)
}

al. solved Maxwell's equations for transferring waves on earth, using Yee's method and FVM [6]. Lebedev et al. discussed a second order accurate FVM [7]. Another popular method for unstructured grids is Finite Element Method (FEM) [8]. FEM is highly accurate, but very complicated. Moreover, its computational cost is very high [9].

Although FVM and FEM can be used for unstructured grids, because of their complexity and high computational cost, FDM is preferred. In order to make this method applicable to unstructured grids, based on Yee's method, a new method is introduced for the unstructured grids [10-12]. The problem with this method is that, in addition to the main grid, a secondary grid, normal to the first grid, should be constructed. Consequently, this method is complicated, and because of this dual mesh, computational cost is high.

In this paper, based on Yee's method, a new approach for attaining a numerical solution to Maxwell's equations for the sinusoidal steady-state case on unstructured grids is introduced. This numerical approach is capable to be applied to a single unstructured grid, which makes it simpler and less expensive than the dual mesh methods. In the following sections, first, the governing equations are specified and, then, 
the numerical method is introduced. Finally, by comparison of numerical and analytical solutions to a specific problem, the numerical approach is validated.

\section{Governing equations}

Maxwell's equations consist of two vectors and two scalar equations. Derivative forms of Maxwell's equations in an isotropic environment are [13]:

$$
\begin{aligned}
& \epsilon \nabla \cdot \vec{E}=\rho, \\
& \nabla \cdot \vec{H}=0, \\
& \nabla \times \vec{E}=-\mu \frac{\partial \vec{H}}{\partial t}, \\
& \nabla \times \vec{H}=\epsilon \frac{\partial \vec{E}}{\partial t}+\vec{J} .
\end{aligned}
$$

In these equations, $\epsilon$ is permittivity and $\mu$ is permeability of the environment. $\vec{E}, \vec{H}$, and $\vec{J}$ are electric field vector, magnetic field intensity vector, and electric current density vector, respectively, which generally depend on both the location and time. In most cases, Maxwell's equations should be solved numerically. There are only 6 unknowns in these equations, namely, 3 components of electric field and 3 components of magnetic field. Therefore, to obtain the results, the solution to Vector Eqs. (3) and (4) suffices. Based on Eqs. (3) and (4), in an enclosed environment, if the input current is harmonic in time, after damping the unsteady terms, the solution will also be harmonic in time. Therefore, the forms of electric current density, electric field, and magnetic field intensity are:

$$
\begin{aligned}
& \vec{J}(\vec{r}, t)=\overrightarrow{\tilde{J}}(\vec{r}) \sin (\omega t), \\
& \vec{E}(\vec{r}, t)=\overrightarrow{\tilde{E}}(\vec{r}) \cos (\omega t), \\
& \vec{H}(\vec{r}, t)=\overrightarrow{\tilde{H}}(\vec{r}) \sin (\omega t) .
\end{aligned}
$$

In these equations, $\omega$ is the variation frequency of the current density. By substituting the above equations in Eqs. (1) to (4) and simplifying, the amplitude's equations are specified as

$$
\begin{aligned}
& \nabla \cdot \overrightarrow{\tilde{E}}(\vec{r})=0, \\
& \nabla . \overrightarrow{\tilde{H}}(\vec{r})=0, \\
& \nabla \times \overrightarrow{\tilde{E}}(\vec{r})=-\mu \omega \overrightarrow{\tilde{H}}(\vec{r}), \\
& \nabla \times \overrightarrow{\tilde{H}}(\vec{r})=-\epsilon \omega \overrightarrow{\tilde{E}}(\vec{r})+\overrightarrow{\tilde{J}}(\vec{r}) .
\end{aligned}
$$

If the current is periodic but not harmonic, since the Maxwell's equations are linear, it is possible to solve Eqs. (8) to (11) for each component of the Fourier series expansion of the current and then, add the component solutions to obtain the final solution.

It is more convenient to work with dimensionless form of the equations.

Therefore, dimensionless variables can be defined as:

$$
\begin{aligned}
& \vec{r}=\frac{\vec{r}}{L}, \quad \vec{J}(\vec{r})=\frac{\overrightarrow{\tilde{J}}(\vec{r})}{\hat{J}}, \\
& \vec{E}(\vec{r})=\frac{\overrightarrow{\tilde{E}}(\vec{r})}{\mu \omega L^{2} \hat{J}}, \quad \vec{H}(\vec{r})=\frac{\overrightarrow{\tilde{H}}(\vec{r})}{L \hat{J}} .
\end{aligned}
$$

where $L$ is a length scale, such as the order of the chamber's dimensions, and $\hat{J}$ is the order of the imposed electric current density. By applying these variables to Eqs. (8) to (11), the dimensionless forms of these equations are obtained as:

$$
\begin{aligned}
& \nabla \cdot \vec{E}=0, \\
& \nabla \cdot \vec{H}=0, \\
& \nabla \times \vec{E}=-\vec{H}, \\
& \nabla \times \vec{H}=-\left(\frac{\omega L}{c}\right)^{2} \vec{E}+\overrightarrow{\vec{J}}
\end{aligned}
$$

In Eq. (16), $c=\frac{1}{\sqrt{\epsilon \mu}}$ is the light velocity in the environment.

\section{Numerical approach}

By integrating Eqs. (13) to (16) in a control volume, the integral forms of the equations are specified as:

$$
\begin{aligned}
& \int_{S} \vec{E} \cdot \overrightarrow{d s}=0 \\
& \int_{S} \vec{H} \cdot \overrightarrow{d s}=0 \\
& \oint_{\Gamma} \vec{E} \cdot \overrightarrow{d l}=-\int_{S} \vec{H} \cdot \overrightarrow{d s} \\
& \oint_{\Gamma} \vec{H} \cdot \overrightarrow{d l}=\int_{S}\left(-\left(\frac{\omega L}{c}\right)^{2} \vec{E}+\vec{J}\right) \cdot \overrightarrow{d s} .
\end{aligned}
$$

By applying Eqs. (17) and (18) to a tetrahedral mesh and using the mean amount of fields on the surfaces 
of each volume, the numerical continuity equations can be found as:

$$
\begin{aligned}
& \sum_{i=1}^{4} \dot{E}_{i, n} A_{i}=0 \\
& \sum_{i=1}^{4} \dot{H}_{i, n} A_{i}=0 .
\end{aligned}
$$

In these equations, $A_{i}$ is area of the $i$ th surface on a given volume, and $\dot{E}_{i, n}$ and $\dot{H}_{i, n}$ are the mean normal components of $E^{\prime}$ and $H^{\prime}$ on that surface, respectively.

By applying Eqs. (19) and (20) to the triangular surfaces of the mesh, and using the mean amount of fields on the edges of the surfaces, the numerical curl equations can be determined as:

$$
\begin{aligned}
& \sum_{i=1}^{3} \dot{E}_{i, l} L_{i}=-\dot{H}_{n} A \\
& \sum_{i=1}^{3} \dot{H}_{i, l} L_{i}=\left(-\left(\frac{\omega L}{c}\right)^{2} \dot{E}_{n}+\dot{J}_{n}\right) A .
\end{aligned}
$$

In these equations, $L_{i}$ is length of the $i$ th edge on a given surface, $A$ is area of that surface, and $\dot{E}_{i, l}$ and $\dot{H}_{i, l}$ are the mean parallel components of $E^{\prime}$ and $H^{\prime}$ on the edge, respectively. The positive direction of the fields on the edges should follow the right-hand thumb rule.

The suggested algorithm for attaining a numerical solution to Maxwell's equations is shown in Figure 1 and summarized as follows:

1. By using the initial amount of electric field and current density on the nodes, their normal vectors on the surfaces of the grid are determined by averaging.

2. By using these normal amounts of electric field and current density, the right-hand side of Eq. (24) is calculated for all grid surfaces. Then, this equation should be numerically solved for all surfaces. For attaining the numerical solution to this equation, an iterative method is applied. It means that for every surface, the fields on the left-hand side of Eq. (24) change in a way that this equation is satisfied for those surfaces. It means that the edges field should change by Eq. (25).

$$
\dot{H}_{i, l, \text { new }}=\dot{H}_{i, l}+\frac{(\mathrm{RHS} \text { of Eq. }(24))-\sum_{i=1}^{3} \dot{H}_{i, l} L_{i}}{\sum_{i=1}^{3} L_{i}} .
$$

The index "new" indicates the new amount of magnetic field obtained through iteration. Iterations continue until convergence is reached.

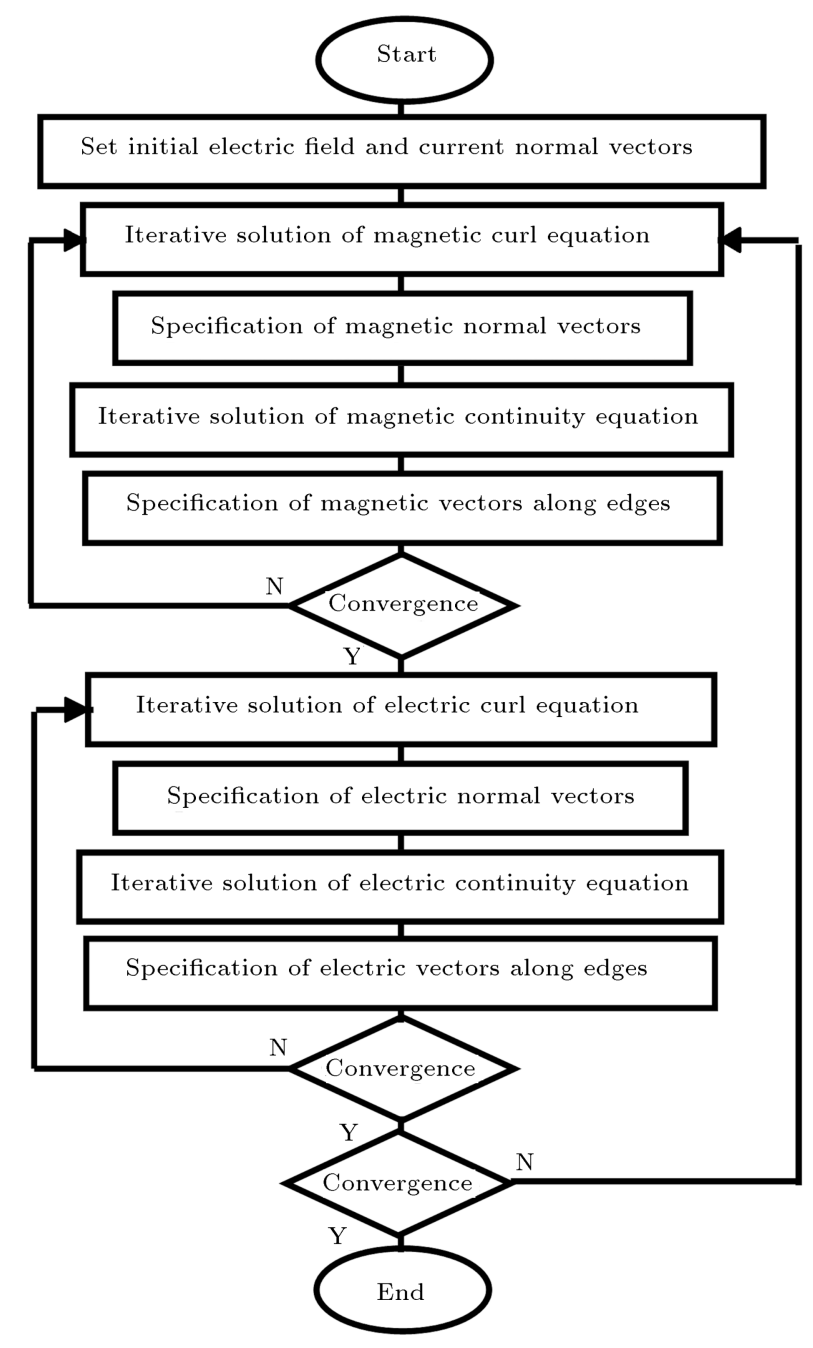

Figure 1. Suggested algorithm for attaining numerical solution to sinusoidal steady-state Maxwell's equations.

3. By new amount of magnetic field along the edges, new amounts of magnetic field on the nodes and, then, normal to the surfaces are specified.

4. In order to increase accuracy of the result, the continuity equation for magnetic field in Eq. (22) must be satisfied. Similar to the method determined in stage 2, continuity equation must be satisfied iteratively.

In this case, new amounts of normal vectors should be changed in a way that continuity equation for the volume is satisfied. Thus, the following relation should be used:

$$
\dot{H}_{i, n, \text { new }}=\dot{H}_{i, n}-\frac{\sum_{i=1}^{4} \dot{H}_{i, n} A_{i}}{\sum_{i=1}^{4} A_{i}} .
$$

Again, iterations continue until convergence is reached. 
5. By new amounts of magnetic field normal to the surfaces, new amounts of magnetic field on the nodes and, then, along the edges are specified.

6 . Stages 2 to 5 should be repeated until the results converge and satisfy both Eqs. (22) and (24).

7. Having normal vector of magnetic field obtained through previous stages, the right-hand side of Eq. (23) is calculated. Then, this equation should be solved similar to Eq. (24) by the method that was mentioned in stage 2 .

8. Normal vectors of the electric field on the surfaces are specified as in stage 3 .

9. Again, to increase the accuracy, the continuity equation for electric field in Eq. (21) should be satisfied. This is done as in the method determined in stage 4 .

10. Similar to stage 5 , by new amounts of electric field normal to the surfaces, new amounts of electric field on the nodes and, then, along the edges are determined.

11. Stages 7 to 10 should be repeated until the results converge and satisfy both Eqs. (21) and (23).

12. Having new amounts of electric field in the environment, stages 2 to 11 should be repeated until the results become convergent and satisfy Eqs. (21) to $(24)$, simultaneously.

\section{Validation of the method}

In order to validate the suggested method, a problem whose analytical solution is available will be numerically solved and the results will be compared with the analytical solution. A schematic of the problem is provided in Figure 2. A non-metal straight wire with radius of 0:1 (dimensionless units), located in the center of a cylinder with radius of $1: 5$ and height of 2 , conducts a sinusoidal current with maximum amount of 1 . The

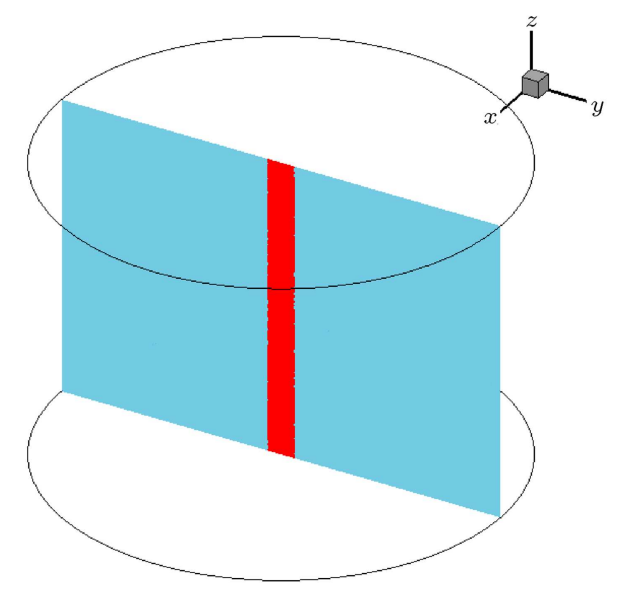

Figure 2. The considered problem for validation of the method. materials of the wire and the surrounding environment are considered to be different so that $\frac{\omega L}{c}$ for wire is 1 and for surrounding environment is $0: 1$. The wall of the chamber is metallic, so normal magnetic field and parallel electric field on the wall are zero.

The analytical solution to this problem is (the details are discussed in the appendix):

$$
\begin{aligned}
& \left\{\begin{array}{cc}
\dot{E}_{z}=1-1.0166 J_{0}(\dot{r}) & \dot{r} \leq 0.1 \\
E_{z}=\begin{array}{l}
0.0041 J_{0}(0.316 \dot{r}) \\
+0.0080 Y_{0}\left(0.316 r^{\prime}\right)
\end{array} & \dot{r}>0.1
\end{array}\right. \\
& \left\{\begin{array}{rr}
\dot{H}_{\theta}=1.0166 J_{1}(\dot{r}) & \dot{r} \leq 0.1 \\
\dot{H}_{\theta}=-0.0013 J_{1}\left(0.316 \dot{r}^{\prime}\right) & \\
-0.0025 Y_{1}\left(0.316 \dot{r}^{\prime}\right) & \dot{r}>0.1
\end{array}\right.
\end{aligned}
$$

where $J_{0}, J_{1}, Y_{0}$, and $Y_{1}$ are Bessel functions. This problem is also numerically solved by the suggested numerical method. Figure 3 shows the grid used for the numerical solution. This grid consists of about 48000 nodes that are distributed with higher density around the surface of the wire where an acute change in the solution is expected. The same simulation is performed on two more resolved grids with the numbers of nodes of about 179000 and 312000 without any significant change in the results, which means the solution is accurate enough with respect to the grid resolution.

Although the problem has axial and rotational symmetries, the numerical method does not exploit these symmetries. In other words, since the grid is unstructured, the numerical domain is not symmetric, and the symmetries and simplicity of the problem do not affect the numerical procedure. Therefore, a simple cylindrical geometry is used for validation of the method because of two reasons: first, the simplicity of the problem is not relevant to the numerical method and, second, there is an analytical solution to this problem that can be referred to for validation.

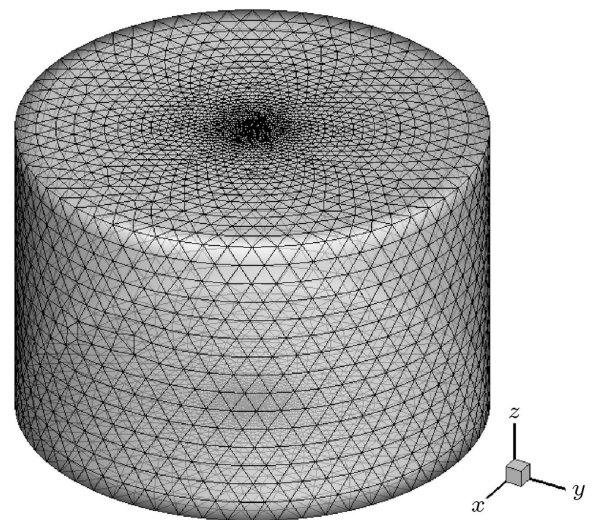

Figure 3. The grid used for numerical solution to the case problem. 


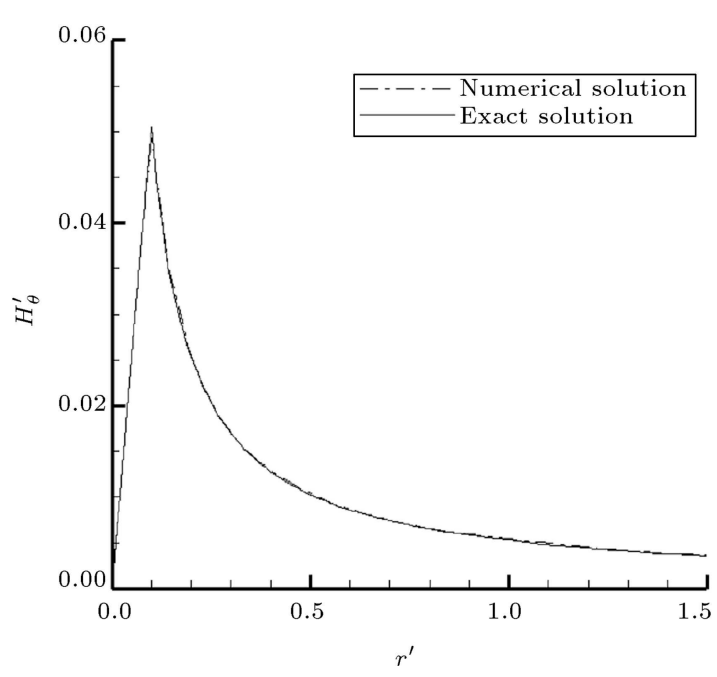

Figure 4. Comparison of the analytical and numerical solutions. The analytical and numerical solutions are very close to each other and it is difficult to distinguish them.

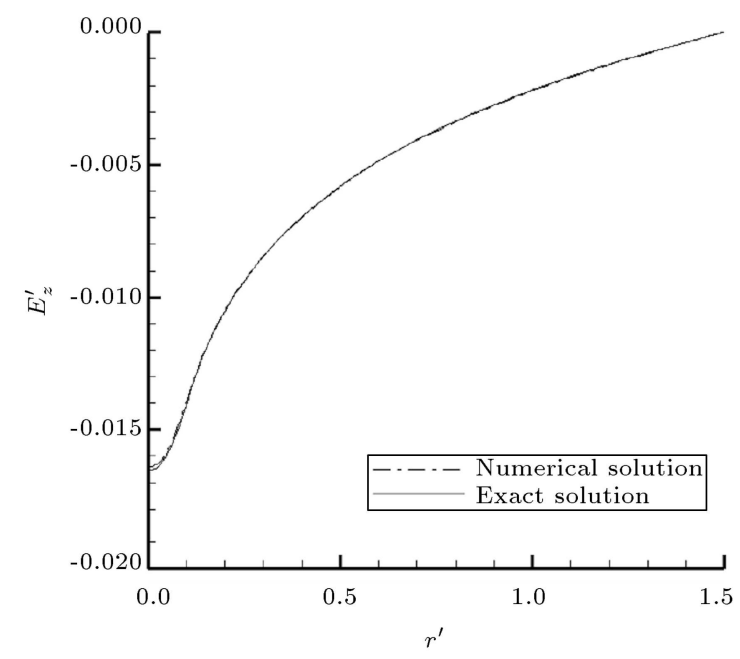

Figure 5. Comparison of the analytical and numerical solutions. The analytical and numerical solutions are very close to each other and it is difficult to distinguish them.

In Figures 4 and 5, the analytical and numerical solutions are compared. Based on these figures, the numerical solution is very close to the analytical solution. Since the grid is unstructured, simplicity of the problem does not affect the numerical solution. Therefore, it is concluded that the numerical approach is accurate enough, and can be used to solve Maxwell's equations.

\section{Conclusion}

A heuristic approach to numerically solve Maxwell's equations was suggested. This method was based on Yee's method, but could be applied to unstructured grids. This paper proposed the method for attaining a numerical solution to sinusoidal steady-state prob- lems, but it is also possible to apply this method to fully unsteady problems. For unsteady problems, the suggested algorithm should be utilized in every time step. Further investigation could be done to increase the accuracy of the method by improvement of the transferring and averaging mechanisms.

\section{Nomenclature}

$\dot{E}_{\theta} \quad \theta$-component of $E^{\prime}$

$\dot{E}_{n} \quad$ Mean normal component of $\dot{E}$ on a given surface

$E_{r} \quad r$-component of $E^{\prime}$

$\dot{E}_{z} \quad z$-component of $E^{\prime}$

$\dot{E}_{i, l} \quad$ Mean parallel component of $\dot{E}$ on the $i$ th edge of a given surface

$\dot{E}_{i, n} \quad$ Mean normal component of $\dot{E}$ on the $i$ th surface of a given volume

$\dot{H}_{\theta} \quad \theta$-component of $H^{\prime}$

$\dot{H}_{n} \quad$ Mean normal component of $\dot{H}$ on a given surface

$\dot{H}_{r} \quad r$-component of $H^{\prime}$

$\dot{H}_{z} \quad z$-component of $H^{\prime}$

$\dot{H}_{i, l} \quad$ Mean parallel component of $\dot{E}$ on the $i$ th edge of a given surface

$\dot{H}_{i, n} \quad$ Mean normal component of $\dot{H}$ on the $i$ th surface of a given volume

$\dot{J}_{n} \quad$ Mean normal component of $\dot{J}$ on a given surface

$\epsilon \quad$ Permittivity $\left(\mathrm{C}^{2} \cdot \mathrm{N}^{-1} \cdot \mathrm{m}^{-2}\right)$

$\hat{J} \quad$ Order of imposed electric current density into the chamber

$\mu \quad$ Permeability $\left(\right.$ N.A $\left.{ }^{-2}\right)$

$\omega \quad$ Fields variation frequency $\left(\mathrm{rad}_{\mathrm{s}} \mathrm{s}^{-1}\right)$

$\rho \quad$ Electric charge volume density $\left(\mathrm{C} . \mathrm{m}^{-3}\right)$

$\vec{E} \quad$ Dimensionless amplitude of electric field vector

$Y_{1} \quad$ First order Bessel function of the second kind

$\vec{H} \quad$ Dimensionless amplitude of magnetic field intensity vector

$\vec{j} \quad$ Dimensionless amplitude of electric current density vector

$\vec{r} \quad$ Dimensionless location vector

$\overrightarrow{\tilde{E}} \quad$ Amplitude of electric field vector $\left(\mathrm{V} . \mathrm{m}^{-1}\right)$ 
Amplitude of magnetic field intensity vector $\left(\right.$ A.m $\left.{ }^{-1}\right)$

Amplitude of electric current density vector $\left(\right.$ A.m $\left.{ }^{-2}\right)$

$\vec{E} \quad$ Electric field vector $\left(\mathrm{V} . \mathrm{m}^{-1}\right)$

$\vec{H} \quad$ Magnetic field intensity vector $\left(\right.$ A.m $\left.{ }^{-1}\right)$

$\vec{J} \quad$ Electric current density vector $\left(\right.$ A.m $\left.{ }^{-2}\right)$

$\vec{r} \quad$ Location vector $(\mathrm{m})$

$A_{i} \quad$ Non-dimensional area of the $i$ th surface of a given volume

c $\quad$ Light velocity $\left(\mathrm{m} . \mathrm{s}^{-1}\right)$

$J_{0} \quad$ Zero order Bessel function of the first kind

$J_{1} \quad$ First order Bessel function of the first kind

$L \quad$ Length scale of the chamber

$L_{i} \quad$ Non-dimensional length of the $i$ th edge of a given surface

$t \quad$ Time (s)

$Y_{0} \quad$ Zero order Bessel function of the second kind

\section{Acknowledgement}

The authors would like to thank Dr. Asghar Molayi Dehkordi and Dr. Mehrdad Taghizadeh Manzari for their comments on the method suggested in this paper.

\section{References}

1. Yee, K. "Numerical solution of initial boundary value problems involving Maxwell's equations in isotropic media", IEEE Transactions on Antennas and Propagation, 14(3), pp. 302-307 (1966).

2. Taflove, A. "Application of the finite-difference time-domain method to sinusoidal steady-state electromagnetic-penetration problems", IEEE Transactions on Electromagnetic Compatibility, 3, pp. 191-202 (1980).

3. Taflove, A. and Umashankar, K. "Radar cross section of general three-dimensional scatterers", IEEE Transactions on Electromagnetic Compatibility, 4, pp. 433440 (1983).

4. Inan, U.S. and Marshall, R.A., Numerical Electromagnetics: The FDTD Method, Cambridge University Press (2011).

5. Shang, J.S. "Characteristic-based algorithms for solving the Maxwell equations in the time domain", IEEE Antennas and Propagation Magazine, 37(3), pp. 15-25 (1995).
6. Jahandari, H. and Farquharson, C.G. "A finite-volume solution to the geophysical electromagnetic forward problem using unstructured grids", Geophysics, 79(6), pp. E287-E302 (2014).

7. Lebedev, A.S., Fedoruk, M.P., and Shtyrina, O.G.V. "Finite-volume algorithm for solving the time-dependent Maxwell equations on unstructured meshes", Computational Mathematics and Mathematical Physics, 46(7), pp. 1219-1233 (2006).

8. Fahs, H., Fezoui, L., Lanteri, S., and Rapetti, F. "Preliminary investigation of a nonconforming discontinuous Galerkin method for solving the time-domain Maxwell equations", IEEE Transactions on Magnetics, 44(6), pp. 1254-1257 (2008).

9. Um, E.S., Harris, J.M., and Alumbaugh, D.L. "3D time-domain simulation of electromagnetic diffusion phenomena: A finite-element electric-field approach", Geophysics, 75(4), pp. F115-F126 (2010).

10. Bossavit, A. and Kettunen, L. "Yee-like schemes on staggered cellular grids: A synthesis between FIT and FEM approaches", IEEE Transactions on Magnetics, 36(4), pp. 861-867 (2000).

11. Bossavit, A. and Kettunen, L. "Yee-like schemes on a tetrahedral mesh, with diagonal lumping", International Journal of Numerical Modelling Electronic Networks Devices and Fields, 12, pp. 129-142 (1999).

12. Sazonov, I., Hassan, O., Morgan, K. and Weatherill, N.P. "Yee's scheme for the integration of Maxwell's equation on unstructured meshes", In ECCOMAS CFD 2006: Proceedings of the European Conference on Computational Fluid Dynamics, Egmond aan Zee, The Netherlands, September 5-8, 2006, Delft University of Technology; European Community on Computational Methods in Applied Sciences (ECCOMAS) (2006).

13. Lehner, G., Electromagnetic Field Theory for Engineers and Physicists, Springer Science \& Business Media (2010).

\section{Appendix}

Analytical solution to the considered problem First, the problem of a wire with infinite length located in the center of a cylinder is considered. Because of the symmetry in the problem, the $\theta$-direction and $z$-direction derivatives are zero, and the fields only depend on $r$-direction. As a result, Eqs. (15) and (16) yield:

$$
\begin{aligned}
& 0=-\dot{H}_{r}, \\
& -\frac{\partial \dot{E}_{z}}{\partial \dot{r}}=\dot{H}_{\mathrm{heta}}, \\
& \frac{1}{\dot{r}} \frac{\partial}{\partial \dot{r}}\left(\dot{r}^{\prime} \dot{E}_{\theta}\right)=-\dot{H}_{z}, \\
& 0=-\left(\frac{\omega L}{c}\right)^{2} \dot{E}_{r},
\end{aligned}
$$




$$
\begin{aligned}
& -\frac{\partial \dot{H}_{z}}{\partial \dot{r}}=-\left(\frac{\omega L}{c}\right)^{2} \dot{E}_{\theta}, \\
& \% \frac{1}{\dot{r}} \frac{\partial}{\partial \dot{r}}\left(\dot{r} \dot{H}_{\theta}\right)=-\left(\frac{\omega L}{c}\right)^{2} \dot{E}_{z}+\dot{J}_{z},
\end{aligned}
$$

Based on Eqs. (A.1) and (A.4), the $r$-components of the fields are zero. Boundary conditions for the problem are:

$$
\begin{aligned}
& \begin{cases}\dot{E}_{1, \theta}=\text { bounded } & \dot{r}=0 \\
\dot{E}_{1, \theta}=\dot{E}_{2, \theta}, \dot{H}_{1, z}=H_{2, z} & \dot{r}=0.1 \\
\dot{E}_{2, \theta}=0 & \dot{r}=1.5\end{cases} \\
& \begin{cases}\dot{E}_{1, z}=\text { bounded } & \dot{r}=0 \\
\dot{E}_{1, z}=\dot{E}_{2, z}, \dot{H}_{1, \theta}=\dot{H}_{2, \theta} & \dot{r}=0.1 \\
\dot{E}_{2, z}=0 & \dot{r}=1.5\end{cases}
\end{aligned}
$$

In these equations, the index 1 indicates the location inside the wire and the index 2 indicates outside of the wire. Based on Eqs. (A.3), (A.5), and the boundary condition (Eq. (A.7)), the $\theta$-component of the electric field and the $z$-component of magnetic field are zero. Combining Eqs. (A.2) and (A.6) gives the final equation for $z$-component of electric field:

$$
\dot{r}^{2} \frac{\partial^{2} \dot{E}_{z}}{\partial \dot{r}^{2}}+\dot{r} \frac{\partial \dot{E}_{z}}{\partial \dot{r}}+\left(\frac{\omega L}{c}\right)^{2} \dot{r}^{2} \dot{E}_{z}=\dot{r}^{2} \dot{J}_{z}
$$

This equation is the Bessel differential equation whose analytical solution is available. Solving this equation for wire and the surrounding environment, separately, and using Eq. (A.2) give the analytical solution to the infinite-length wire problem:

$$
\begin{gathered}
\left\{\begin{array}{rr}
\dot{E}_{z}=1-1.0166 J_{0}(\dot{r}) & \dot{r} \leq 0.1 \\
\dot{E}_{z}=0.0041 J_{0}(0.316 \dot{r}) & +0.0080 Y_{0}(0.316 \dot{r})
\end{array}\right. \\
\left\{\begin{array}{rr}
\dot{H}_{\theta}=1.0166 J_{1}(\dot{r}) & \dot{r} \leq 0.1 \\
\dot{H}_{\theta}=-0.0013 J_{1}(0.316 \dot{r}) & \\
-0.0025 Y_{1}(0.316 \dot{r}) & \dot{r}>0.1
\end{array}\right.
\end{gathered}
$$

Since in this problem, $\dot{E}_{\theta}, \dot{E}_{r}$, and $\dot{H}_{z}$ are zero, the solution satisfies the boundary conditions of the considered problem. Thus, the results satisfy Eqs. (15) and (16) and the boundary conditions of the considered problem. Consequently, the results of the case problem are the results of the infinite-length wire problem.

\section{Biographies}

Sajjad Azimi obtained his BS degree in 2012 and MS degree in 2015 in Mechanical Engineering from Sharif University of Technology, Tehran, Iran. He is now a PhD student at École Polytechnique Fédérale de Lausanne, Lausanne, Switzerland. His fields of study are biofluids, microfabrication, and boundary layer flows.

Mohammad Said Saidi received his $\mathrm{PhD}$ degree from Massachusetts Institute of Technology, USA, in 1979, and is currently Professor of Mechanical Engineering at Sharif University of Technology, Tehran, Iran. His research interests include, but are not limited to, CFD, micro and macro multiphase flows in human body, experimental design, and mathematical modeling of transport of nano- and micro-scale aerosol particles. 